\title{
From the Fourth International Conference on the Prevention of Infection
}

\section{Automatic Alerts for Methicillin-Resistant Staphylococcus aureus Surveillance and Control: Role of a Hospital Information System}

\author{
Didier Pittet, MD, MS; Edith Safran, MD; Stephan Harbarth, MD; François Borst, MD; Pascale Copin, RN; \\ Peter Rohner, MD; Jean-Raoul Scherrer, MD; Raymond Auckenthaler, MD
}

\begin{abstract}
BACKGROUND: Methicillin-resistant Staphylococcus aureus (MRSA) is an escalating problem in hospitals worldwide. The hospital reservoir for MRSA includes recognized and unrecognized colonized or infected patients, as well as previously colonized or infected patients readmitted to the hospital. Early and appropriate infection control measures (ICM) are key elements to reduce MRSA transmission and to control the hospital reservoir.

OBJECTIVE: To describe the role of an expert system applied to the control of MRSA at a large medical center $(1,600$ beds) with high endemic rates.

METHODS: The University Hospital of Geneva has an extended hospital information system (HIS), DIOGENE, structured with an open distributed architecture. It includes administrative, medical, nursing, and laboratory applications with their relational databases. Among available patient databases, clinical microbiology laboratory and admission-discharge-transfer (ADT) databases are used to generate computer alerts. A laboratory alert (lab alert) is printed daily in the Infection Control Program (ICP) offices, listing all patients with cultures positive for MRSA detected within the preceding 24 hours. Patients might be either newly detected patients colonized or infected with MRSA, or previously recognized MRSA patients having surveillance cultures. The ICP

nurses subsequently go to the ward or call the ward personnel to implement ICM. A second alert, the "readmission alert," detects readmission to the hospital of any patient previously colonized or infected with MRSA by periodic queries (q $1 \mathrm{~min}$ ) to the ADT database. The readmission alert is printed in the ICP offices, but also forwarded with added guidelines to the emergency room.

RESULTS: During the first 12 months of application (July 1994 to June 1995), the lab alert detected an average of 4.6 isolates per day, corresponding to 314 hospital admissions (248 patients); the use of this alert saved time for the ICP nurses by improving work organization. There were 438 readmission alerts (1.2 alerts per day) over the study period; of 347 patients screened immediately upon readmission, 114 (33\%) were positive for MRSA carriage. Delayed recognition of readmitted MRSA carriers decreased significantly after the implementation of this alert; the proportion of MRSA patients recognized at the time of admission to the hospital increased from $13 \%$ in 1993 to $40 \%$ in $1995(P<.001)$.

CONCLUSIONS: Hospital information system-based alerts can play an important role in the surveillance and early prevention of MRSA transmission, and it can help to recognize patterns of colonization and transmission (Infect Control Hosp Epidemiol 1996;17:496-502).
\end{abstract}

\section{INTRODUCTION}

Methicillin-resistant Staphylococcus aureus (MRSA) has become a worldwide problem, with an important impact on healthcare costs and medical management. ${ }^{1}$ In the United States, hospitals of all sizes are facing an increasing problem of MRSA. 2,3 Some countries, such as Japan, already have been overwhelmed by the MRSA epidemic. In Europe, the prevalence of methicillin resistance among $S$ aureus isolates ranges from less than $1 \%$ in Scandinavia to more than $30 \%$ in France, Spain, and Italy. 4 Low-prevalence countries such as The Netherlands or Belgium try to prevent the escalating problem of MRSA by

From the Infection Control Program (Drs. Pittet and Harbarth, and Ms. Copin), Hospital Information System (Drs. Safran, Borst, and Scherrer), Clinical Microbiology Laboratory (Drs. Rohner and Auckenthaler), University Hospital of Geneva, Geneva, Switzerland.

The authors thank all the participants in this study: A. Alexiou, J. Boissard-Sztajzel, N. Henri, V. Sauvan, S. Touvenau (Infection Control Program); B. Pepey (Clinical Microbiology Laboratory); G. Thurler, P. Schulthess, L. Rebouillat, M. Lagana, J-P. Berney, M. Berthoud (Hospital Information System).

Address reprint requests to Didier Pittet, $M D, M S$, Infection Control Program, Division of Infectious Diseases, Department of Internal Medicine, 24, rue Micheli-du-Crest, University Hospital of Geneva (HCUG), 1211 Geneva 14, Switzerland.

96-OAC-101. Pittet D, Safran E, Harbarth S, Borst F, Copin P, Rohner P, Scherrer J-R, Auckenthaler R. Automatic alerts for methicillinresistant Staphylococcus aureus surveillance and control: role of a hospital information system. Infect Control Hosp Epidemiol 1996;17:496-502. 
portion of patients admitted and readmitted with MRSA: in $1993,17 \%$ of all $S$ aureus isolates in this institution were methicillin-resistant.

Usually, MRSA is introduced into a hospital by an infected or colonized patient. After initial dissemination, MRSA populates human and environmental niches and establishes reservoirs among persons, sometimes including healthcare personnel, who may become colonized and infected. The prevalent cohort of colonized and infected patients generally is assumed to be the most significant reservoir from which MRSA is transmitted to other individuals. ${ }^{9}$ Early and appropriate infection control measures (ICM) are key elements to reduce MRSA transmission and to control the hospital reservoir. $1,10,11$

Computer alerts in medicine are a well-known tool to help notification of medical personnel about a critical action to be taken or other important in-hospital events. 12 One of the first systems used was the Health Evaluation Through Logical Processing (HELP) system at Latter Day Saints Hospital in Salt Lake City, Utah. ${ }^{13}$ Developed since the 1950s, HELP is well known for the implementation of decision logic mechanisms in various applications. In particular, the HELP system is used to generate alerts advising staff of relevant infection surveillance data stored in hospital information system (HIS) databases. ${ }^{14-16}$ Other HISbased computer alerts have been developed since in various fields and applications.

So far, no extensive experience has been published concerning computer alerts for the surveillance and control of MRSA. The objective of the present study is to describe the role of an expert system applied to the control of MRSA at a large medical center.

\section{METHODS Hospital}

The University of Geneva Hospital, Switzerland, is a 1,600-bed healthcare center providing primary and tertiary medical care for Geneva and the surrounding areas ( 1,000,000 population). Approximately 40,000 patients are admitted annually for a mean length of stay of 11 days. The Infection Control Program (ICP), established in October 1992, consists of one hospital epidemiologist, one fellow, and five full-time infection control nurses (ICNs).

\section{Surveillance and Infection Control Measures}

Since 1992, the infection control team has conducted several hospitalwide period-prevalence studies to detect nosocomial infections at HCUG, using total chart surveillance. 17 Collection of data was based on standard definitions. ${ }^{18}$ The ICP team also conducted prospective, warddirected surveillance for nosocomial infections between October 1992 and December 1995, by twice-weekly visits in general wards and daily (except weekends) visits in intensive-care units.

Since January 1993, control measures have been implemented in an attempt to control the spread of MRSA within this institution. All patients colonized or infected with MRSA were followed prospectively from the day of first MRSA identification to the time of discharge from the hospital, by weekly or twice weekly visits. Data collection included patient demographics, underlying illness, comorbidities, dates and sites of colonization and infection, device utilization, exposure to risk factors for MRSA colonization, type of therapy, and outcome. Information for each patient was obtained by nursing and medical chart review, review of laboratory data, clinical examination, and, when necessary, telephone interviews to private physicians or outside hospitals, and clinical examination. A dedicated case report form was used, and data were stored in a specially designed database (Microsoft Access, version 2.0, Microsoft Co, Ireland).

The following ICM were used:

(1) All patients colonized or infected with MRSA were placed on contact isolation, as recommended by Centers for Disease Control and Prevention (CDC) guidelines, ${ }^{19}$ until the time of discharge or until eradication of colonization was documented by two consecutive sets of negative surveillance cultures (separated by at least 24 hours).

(2) Active surveillance cultures for MRSA colonization included weekly screening of the nose, axilla, groin, pressure ulcer, wounds, skin insertion sites of percutaneous devices, and urine (if catheter was present). Since January 1994, routine screening of axilla was omitted, because it did not improve the sensitivity of the surveillance cultures to detect MRSA colonization (data not shown).

(3) All patients previously known to be colonized or infected with MRSA were isolated upon readmission to HCUG, and surveillance cultures were obtained. Two consecutive sets of negative surveillance cultures were required to end contact isolation.

(4) Only patients with documented MRSA infections were treated with intravenous antibiotics (usually vancomycin or teicoplanin). Attempts to eradicate MRSA colonization were undertaken routinely (nasal mupirocin ointment, twice per day for 5 days, and chlorhexidine body wash for 10 days). Preliminary results have been reported recently. 20

(5) Following successful eradication, patients remaining in the hospital underwent surveillance cultures weekly for 4 weeks and then monthly to detect return of colonization.

(6) To detect MRSA colonization and cross-infection, surveillance cultures were obtained from roommates as soon as a patient colonized or infected with MRSA was newly identified. Whenever positive for MRSA, and when feasible, the area of surveillance was widened and outbreak investigation was attempted. Molecular typing of MRSA isolates (using contour homogeneous electric-field gel electrophoresis) was performed only in case of outbreak investigation.

Furthermore, patients colonized or infected with MRSA were identified through a combination of the following surveillance techniques:

(1) Since October 1992, clinical microbiology laboratory results were monitored daily, excluding weekends, for isolates of MRSA at any body site. Laboratory technicians telephoned the ICNs in charge of MRSA surveillance to inform them of every newly identified patient. In March 
1994, the automatic laboratory alert (lab alert) system was implemented (see below), replacing the telephone calls made by the laboratory technicians.

(2) Systematic surveillance cultures at the time of admission to the hospital ward with the highest rate of MRSA colonization or infection (the septic orthopedic ward, 45 beds) were implemented in July 1994 to identify nosocomially acquired infections. This ward was the only area of the hospital where systematic screening on admission was performed on all patients.

\section{Definitions}

Colonization was defined as the growth of MRSA in culture from patients without symptoms, signs, or laboratory results meeting the criteria for nosocomial infection. 18 Whenever asymptomatic, patients with urine cultures positive for MRSA were not considered infected, except when the physician prescribed directed antimicrobial therapy.

Patients were considered to have nosocomial acquisition of MRSA if there was no history of prior MRSA colonization or infection and a previous culture of the site was negative during hospitalization. ${ }^{21}$ As proposed by Jernigan and colleagues, 21 in the absence of previous cultures, patients who were discovered to be colonized or infected with MRSA less than 72 hours after admission were assumed to have been admitted with the organism, unless roommates or other nearby patients were discovered to be MRSA carriers. Patients discovered more than 72 hours after admission were considered to be colonized or infected in our institution, unless medical information suggested otherwise (eg, transfer from another hospital or from a nursing home with a previous documentation of MRSA colonization).

The attack rate was defined as the number of newly detected patients colonized or infected with MRSA each year of the study period (January 1989 through December 1995) divided by the annual number of patients admitted at HCUG.

An ambulatory patient who attended outpatient clinics of the institution and for whom a future admission was planned by recording in the HIS was defined as being in preadmission status.

\section{Microbiology}

$S$ aureus was identified using standard laboratory procedures. $^{22}$ The determination of methicillin resistance was performed by use of an oxacillin $(6 \mu \mathrm{g} / \mathrm{mL})$ salt $(2 \%)$ agar screening plate, according to methods recommended by the National Committee for Clinical Laboratory Standards for disk diffusion testing. ${ }^{23-25}$

\section{Hospital Information System and Alerts}

The HIS at HCUG, Division Informatique de L'Hôpital Cantonal de Genève (DIOGENE), is an open distributed system of several networked machines. 26,27 DIOGENE includes many applications related to the patient or to the hospital. Patient applications include an integrated computerized laboratory system, UNILAB, used for lab ordering, results reporting and distribution, and the admission- discharge-transfer application (ADT). In DIOGENE databases, patients are identified with two numbers, the permanent patient identification number and a billing number that is unique for one patient and one hospital stay. All patient databases share the same patient number, allowing linkage of data from different databases.

The lab alert is generated by a UNIX mechanism implemented on the clinical microbiology laboratory database. $^{28}$ The lab alert system is time-driven. Every day at 1:15 PM, it generates two files that immediately are printed in the ICP offices. One of the printed files, "the positive lab alert," contains patient information from the laboratory database for all cultures positive for MRSA identified in the last 24 hours. The second file lists all surveillance cultures for MRSA that showed no growth in the laboratory during the same period. Information on the alerts includes the patient's identity, care unit, date of laboratory order, date of result validation, and type of specimen.

The "readmission alert" is data-driven; it is implemented by an automatic query once per minute to the HCUG-ADT database. The readmission alert detects admission of any patient who was colonized or infected with MRSA during a prior hospital stay at HCUG. ${ }^{28}$ The alert is printed in the ICP offices within minutes after admission. An alert also is generated in case of patient transfer within the hospital (data not included in the present report) or patient discharge from HCUG. Information sent with the alert includes patient identity; date of admission, transfer, or discharge; and care unit. In case of admission through the hospital emergency treatment center, the alert also is printed there and includes guidelines for the emergency center staff.

\section{Statistical Analysis}

Mean quantitative values were compared using the student's $t$ test and the Wilcoxon Rank Sum Test. The frequencies of qualitative variables were compared using the chi-squared test (with Yates' correction, when necessary) or Fisher's Exact Test. Linear trends were tested using linear regression or the chi-squared test for trend, as previously described. ${ }^{29}$ Statistical analyses were performed using Epi Info Software (Epi Info, version 6.0, CDC, Atlanta, GA) and Systat software (Systat, version 5.2, Systat Inc, Evanston, IL). All tests were two-tailed, and $P<.05$ was considered significant.

\section{RESULTS \\ MRSA Trends at HCUG}

Between 1989 and 1995, there were 270,784 admissions to HCUG, representing a total of 3,164,044 patientdays of care. Over the 7-year study period, MRSA colonization or infection was identified in 1,275 admissions, or $0.47 \%$ of all admissions during that time.

The Figure depicts secular trends in the prevalence of patients colonized or infected with MRSA at HCUG. Importantly, incidence rates of MRSA colonization or infection increased significantly $\left(\mathrm{r}^{2}=0.94, P<.001\right)$ over the 7 years of the study, from 0.05 cases per 100 admissions in 1989 to 
0.81 cases per 100 admissions in 1995. The rate of MRSA colonization or infection increased significantly $\left(\mathrm{r}^{2}=0.92\right.$, $P<.043$ ) from 1989 through 1992 (to 0.62 cases per 100 admissions in 1992), but has remained stable since $\left(r^{2}=0.72\right.$, $P=.25)$. The annual attack rate of MRSA colonization or infection (the number of newly recognized MRSA patients, counting each patient only once) remained stable since 1993 (0.55 \pm 0.05 cases per 100 admissions, range 0.51 to 0.62$)$, corresponding to the time of implementation of ICM.

\section{Rates of Infection}

All hospitalized patients colonized or infected with MRSA were followed prospectively since January 1993. Between January 1993 and December 1995, MRSA caused infections in 352 (42\%) of the 843 patients from whom it was isolated. The frequency distribution of MRSA infections by site is shown in the Table. The overall rate of MRSA infection was 3.06 per 1,000 patient admissions from 1993 through 1995 (0.32 episodes per 1,000 patient-days). The rate of MRSA nosocomial infection was 0.12 episodes per 1,000 patient-days in this period. MRSA was responsible for $10 \%$ to $12 \%$ of all nosocomial infections identified in subsequent hospitalwide period-prevalence studies conducted at HCUG in September 1994 and June 1995. Overall, MRSA was the second leading nosocomial pathogen at HCUG, only surpassed by Escherichia coli.

\section{Laboratory Alert}

The lab alert system for positive MRSA results has functioned since March 1994 at HCUG, and the readmission alert since June 9, 1994. Because the purpose of the present work was to describe the role of both alerts, the first 12 months (July 1, 1994, to June 30, 1995) during which both alerts were running were evaluated.

Between July 1994 and June 1995, a total of 1,692 MRSA isolates were identified by the daily lab alert, representing an average of 4.6 MRSA isolates per day. Those MRSA isolates corresponded to 314 hospital stays of 248 different patients. Thus, the mean number of MRSA isolates per colonized or infected patient was 5.4 (median 3; range, 1 to 39 ).

Because surveillance cultures for MRSA colonization frequently were negative, lab alerts also identified negative results. A total of 10,344 negative surveillance cultures collected during 889 hospital admissions were recorded in the study period. Knowledge of those results was important, because it helped the ICNs in charge of MRSA surveillance to adapt ICM promptly when necessary. The time saving associated with the use of the lab alert system was estimated to be approximately 1.5 to 2 hours per day for the ICNs on duty for MRSA. The working time saved for laboratory technicians who otherwise would have been asked to call the ICNs for positive MRSA cultures can be estimated as 10 to 15 minutes per working day.

\section{Readmission Alert}

The readmission alert was activated each time a patient known to be colonized or infected with MRSA dur-

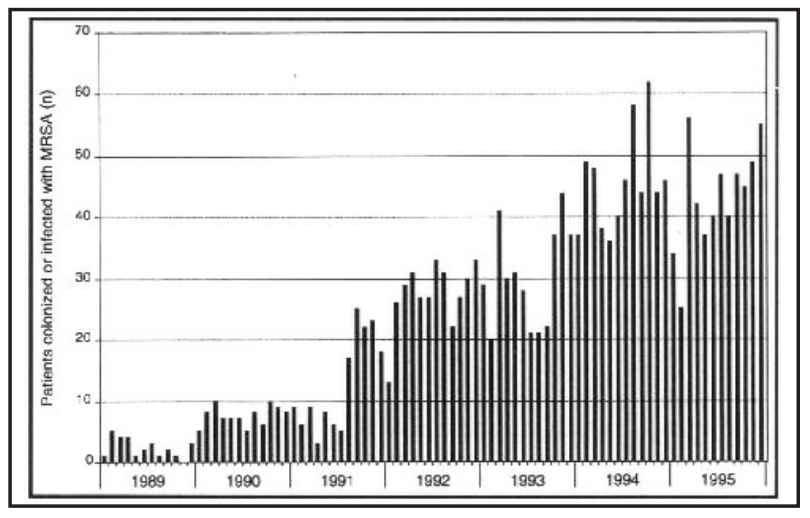

FIGURE. Number of patients colonized or infected with methicillinresistant Staphylococcus aureus at the University Hospital of Geneva, 1989 to 1995, by month and year (prevalent cases).

ing a prior hospital stay was preadmitted, admitted, or discharged. During the 12-month study period, there were a total of 1,314 readmission alerts, with 438 admissions, 306 preadmissions, and 570 discharges. The alerts for discharged patients did not require action, but informed the ICP and helped improve ICNs' work organization.

The 744 admissions or preadmissions represented an average of 2.08 alerts per day (3.2 per working day). Preadmissions of patients previously colonized or infected with MRSA ( $n=306$ ) were flagged to help organize future hospital admission of possibly persistent MRSA carriers; those alerts usually required a telephone call to the wards where the patients were scheduled for admission. There were a total of 438 readmission alerts associated with patient admission to HCUG during the study period, representing an average of 1.2 alerts per day. Among the 438 hospital admissions flagged by the readmission alert, surveillance cultures were performed within the first 24 hours for 347 (79\%). Of the 347 patients screened, 114 (33\%) were positive for MRSA, and 233 (67\%) were negative. Most of the patients identified by the readmission alert who did not undergo surveillance cultures $(n=91)$ had an extremely short length of hospital stay (median 1 day).

Before 1993, the average interval from admission to first screening culture positive for MRSA was 25 days for readmitted patients known to harbor the organism during a previous hospital stay. In 1993, ICM directed toward MRSA were implemented throughout the institution, including a recommendation for labeling of the chart front page. Nonetheless, the delay between admission and surveillance screening for these patients still averaged 8 days (median, 1 day). Following implementation of the readmission alert, previously positive patients were recognized easily upon readmission to HCUG: the time from admission to surveillance cultures averaged 3 days (median, 0 days). Importantly, the proportion of MRSA patients recognized at the time of admission to the hospital increased significantly $(P<.001)$, from $13 \%$ (31 of 235 ) in 1993 to $40 \%$ (124 of 310) in 1995.

Interestingly, the 114 patients with persistent MRSA carriage detected at the time of readmission to HCUG had 
TABLE

Distribution by Site of 401 MRSA Infections AmONG 352 Patients, HCUG, 1993 To 1995

\begin{tabular}{lrc}
\hline & Number & (\%) \\
\hline Surgical site infection & 142 & $(35)$ \\
Urinary tract infection & 109 & $(27)$ \\
Bloodstream infection & 54 & $(13)$ \\
Pneumonia & 33 & $(8)$ \\
Skin/soft tissue infection & 19 & $(5)$ \\
Osteomyelitis/arthritis & 10 & $(3)$ \\
Intraabdominal infection/abscess & 5 & $(1)$ \\
Other & 29 & $(7)$ \\
\hline
\end{tabular}

Abreviations: MRSA, methicillin- resistant Staphylococcus aureus.

HCUG, Hospital Cantonal Universitaire de Genève.

"Other" included intravenous catheter-associated infections, conjunctivitis, meningitis, otitis, and more.

a significantly longer mean hospital stay than did the 233 previously colonized or infected patients with negative surveillance cultures $(P<.05$; mean, 30.4 days; median, 15 days, versus 13.9 and 8 days, respectively).

\section{MRSA Reservoirs}

Of a total of 314 hospital stays of patients colonized or infected with MRSA at HCUG between July 1994 and June 1995, 114 (36\%) were identified immediately upon readmission to the institution by means of the readmission alert. The delay between admission and screening of those patients was minimal (median, 1 day); immediate implementation of ICM was possible for a large majority of those patients (109 of 114, 96\%). None of those patients were responsible for secondary transmission within the institution, despite prolonged hospital stays (mean, 30.4 days; range, 1 to 233 ).

Two hundred additional patients colonized or infected with MRSA were identified through the lab alert system, representing $64 \%$ of the hospital reservoir during the study period. Of those 200, all but two were newly identified colonized or infected MRSA patients. Of the 198 newly identified patients, 163 (82\%) were detected at least 72 hours after admission; however, approximately one third (62 of 163) resulted from nosocomial transmission.

\section{DISCUSSION}

Methicillin-resistant $S$ aureus has emerged as a common nosocomial organism in our hospital and was the second leading pathogen in two consecutive hospitalwide prevalence surveys for nosocomial infections conducted in 1994 and 1995. According to Boyce, 30 HCUG has been a high-endemic-rate institution for MRSA since 1992, with at least 0.5 cases per 100 hospital admissions in this large teaching institution. Rates of MRSA admissions increased linearly between 1989 and 1992, but have remained stable since, possibly due to the implementation of ICM at the end of 1992.

There are two major mechanisms for introduction of MRSA into an institution, the most common being the admission of infected or colonized patients who serve as a reservoir. 9,31,32 The principal mode of MRSA transmission within the institution is from patient to patient via the transiently colonized hands of hospital personnel who acquire the organism after direct patient contact.33 Chronically colonized healthcare workers can disseminate the organism directly, but such occurrence is less common. 33,34 Several studies examined the effect of isolation measures to control nosocomial transmission of MRSA and found a decrease in the incidence of endemic MRSA infection and colonization after adopting barrier isolation precautions. 9,33,35,36 Early implementation of ICM obviously is recommended, and laboratory alert systems may help in this regard.

In endemic or outbreak settings, surveillance is required to determine the degree and extent of the hospital reservoir. The presence of MRSA usually is discovered via routine cultures of infections from hospitalized patients; but routine cultures identify only approximately one third of the hospitalized patients harboring the organism. $9,31,37,38$ An additional one third of the MRSA hospital reservoir can be identified through an admission identification list of all patients harboring the organism during a previous admission or outpatient visit. 33 The final one third of MRSA patients can be detected by cultures of frequently colonized body sites (ie, wounds, tracheostomy sites, tracheal aspirates from intubated patients, etc) from asymptomatic patients, 37 as well as from roommates of newly identified colonized or infected patients.

Our study confirms and extends those findings: $36 \%$ of the hospital reservoir of MRSA patients at HCUG between July 1994 and June 1995 was recognized at the time of admission by the use of the readmission alert. In the study by Lugeon et al, 39 80\% of all episodes of MRSA colonization or infection identified over a 4 -year period were considered hospital acquired, but developed before the implementation of ICM. Delay in implementing those measures is associated with increased risk for MRSA spread to other patients; readmission alert systems can contribute significantly to reductions in delays. Furthermore, MRSA carriage was confirmed in 33\% of patients screened upon readmission in our study; based on these data, immediate implementation of ICM upon readmission, without definitive proof of current MRSA carriage, appears reasonable. Lack of similar information in the literature prevents comparison of our data with those of others.

Attempts to use the computer in medical diagnosis have been mostly unsatisfactory; nevertheless, there is a steady increase in the use of computers as support tools for clinical decisions ${ }^{40-45}$ or infection control. 14-16,46 Since 1994, we have used the automated lab and readmission alert system, which facilitates the investigation and control of the spread of MRSA in our institution. The lab alert is particularly useful for work organization and patient follow-up, whereas the role of the readmission alert is oriented more toward early prevention. Both alerts also are useful tools for diagnosis, treatment, teaching, and quality assessment. This alert system has replaced the slow, time-consuming information system that was based on personal communication to the ICNs after identification of a new or readmitted MRSA- 
patient. Furthermore, the alert system helps greatly to reduce the time to detect and isolate a new or readmitted MRSA patient. Thus, our system is a time-saving tool for our program.

Some limitations of computer alert systems should be mentioned. First, the development and use of automated hospital database and alert systems is costly, and no analysis of the cost-effectiveness has been performed in our institution so far. The combination of isolation precautions, extended screening, and mupirocin or systemic antibiotic treatment of MRSA carriers have been reported to be efficacious $21,37,38,47$ and cost-effective. 21,38 Nevertheless, the timely computer alerting of MRSA patients is likely to contribute to substantial cost savings by preventing early, uncontrolled MRSA transmission and by providing a substantial time saving for the ICP. Second, the protection of individual patient records might be difficult in some circumstances; data from a computer alert system should not be easily accessible for persons outside the ICP. Third, our study results are limited by the lack of systematic microbiological typing for all MRSA isolates.

Finally, we want to underline that computer alert systems are only a tool to help the ICP. We endorse the "shoeleather" approach of the hospital epidemiologist recently described by Weems in this journal. ${ }^{48}$ This approach emphasizes the field activities of the infection control investigator: "pounding the pavement, tracking the evidence, conducting face-to-face interviews, putting one's nose into the problem, and using common sense to complement statistical analysis of data." 48

Nevertheless, in our experience, a computer system to alert and assist in performing surveillance and detection of MRSA-colonization and infection in hospital patients is a useful tool. We recently expanded computer alerts to include surveillance of other nosocomial pathogens (Stenotrophomonas maltophilia, Serratia marcescens, vancomycin-resistant enterococci) and nosocomial bloodstream infections. As hospital information systems improve, this kind of alert system offers great promise for the improved surveillance and control of nosocomial pathogens.

\section{REFERENCES}

1. Wenzel RP, Nettleman MD, Jones RN, Pfaller M. Methicillinresistant Staphylococcus aureus: implications for the 1990s and effective control measures. Am J Med 1991;91:221S-227S.

2. Boyce JM. Increasing prevalence of methicillin-resistant Staphylococcus aureus in the United States. Infect Control Hosp Epidemiol 1990;11:639-642.

3. Panlilio AL, Culver DH, Gaynes RP, et al. Methicillin-resistant Staphylococcus aureus in US hospitals, 1975-1991. Infect Control Hosp Epidemiol 1992;13:582-586.

4. Voss A, Milatovic D, Wallrauch-Schwarz C, Rosdahl VT, Braveny I. Methicillin-resistant Staphylococcus aureus in Europe. Eur J Clin Microbiol Infect Dis 1994;13:50-55.

5. Vandenbroucke-Grauls CMJE. Control of MRSA in The Netherlands. Proceedings of the fourth CIPI. Nice, France. 1996;:S1703. Abstract 70.

6. Struelens MJ, Mertens R, le Groupement pour le Dépistage de MRSA. National survey of methicillin-resistant Staphylococcus aureus in Belgian hospitals: detection methods, prevalence trends, and infection control measures. Eur J Clin Microbiol Infect Dis 1994;13:56-63.
7. Comité de rédaction. Staphylocoques dorés résistants à la méticilline: situation et enjeux. Swiss Noso 1995;2:25-29.

8. Pittet D, Francioli P, von Overbeck J, Raeber PA, Ruef C, Widmer AF. Infection control in Switzerland. Infect Control Hosp Epidemiol 1995;16:49-56.

9. Thompson RL, Cabezudo I, Wenzel RP. Epidemiology of nosocomial infections caused by methicillin-resistant Staphylococcus aureus. Ann Intern Med 1982;97:309-317.

10. Stamm AM, Long MN, Belcher B. Higher overall nosocomial infection rate because of increased attack rate of methicillinresistant Staphylococcus aureus. Am J Infect Control 1993;21:7074.

11. Layton MC, Hierholzer WJ, Patterson JE. The evolving epidemiology of MRSA at a university hospital. Infect Control Hosp Epidemiol 1995;16:12-17.

12. Shortliffe EH. The adolescence of AI in medicine: will the field come of age in the '90s? Art Intelligence Med 1993;5:93-106.

13. Pryor TA, Gardner RM, Clayton PD, Warner HR. The HELP system. J Med Syst 1983;7:87-102.

14. Evans RS, Larsen RA, Burke JP, et al. Computer surveillance of hospital-acquired infections and antibiotic use. JAMA 1986;256:1007-1011.

15. Burke JP, Classen DC, Pestotnik SL, Evans RS, Stevens LE. The HELP system and its application to infection control. J Hosp Infect 1991;18(suppl):424-431.

16. Evans RS, Burke JP, Classen DC, et al. Computerized identification of patients at high risk for hospital-acquired infection. Am J Infect Control 1992;20:4-10.

17. Haley RW, Gaynes RP, Aber RC, Bennett JV. Surveillance of nosocomial infections. In: Bennett JV, Brachman PS, eds. Hospital Infections. 3rd ed. Boston, MA: Little, Brown and Co; 1992:79-108.

18. Garner JS, Jarvis WR, Emori TG, Toran TC, Hughes JM. CDC definition for nosocomial infections. Am I Infect Control 1988;16:128-140.

19. Centers for Disease Control. Guidelines for isolation precautions in hospitals. Infect Control 1983;4 (suppl):245-325.

20. Harbarth S, Pittet D, Copin P, Alexiou A, Henry N, Auckenthaler R. MRSA carriage and infections after one year of institutionalized infection control without use of systemic antimicrobials for eradication of MRSA colonization. Proceedings of the fourth CIPI. Nice, France. 1996:16. Abstract ICN-AMR

21. Jernigan JA, Clemence MA, Stott GA, et al. Control of methicillin-resistant Staphylococcus aureus at a university hospital: one decade later. Infect Control Hosp Epidemiol 1995;16:686696.

22. Kloos WE, Lambe DW. Staphylococcus. In: Balows A, Hausler WJ, Hermann KL, Isenberg HD, Shadomy HJ, eds. Manual of Clinical Microbiology. Washington, DC: American Society of Microbiology; 1991:222-237.

23. National Committee on Clinical Laboratory Standards. (NCCLS document M2A5). Performance Standard for Antimicrobial Disk Susceptibility Tests. Villanova, PA: NCCLS; 1993:14.

24. National Committee on Clinical Laboratory Standards. Methods for dilution antimicrobial susceptibility tests for bacteria that grow aerobically. Villanova, PA: NCCLS; 1993.

25. Baker CN, Huang MB, Tenover FC. Optimizing testing of methicillin-resistant Staphylococcus species. Diagn Microbiol Infect Dis 1994;19:167-170.

26. Scherrer JR, Baud R, Brisebarre A, et al. A hospital information system in continuous operation and expansion. In: Orthner HF, Blum BI, eds. Implementing Healthcare Information Systems. Proceedings of the 10th Symposium on Computer Applications in Medical Care. 1986;100-122. Abstract.

27. Scherrer JR, Baud R, de Roulet D. Moving towards the future design of HIS: a view from the seventies to the end of the nineties, the DIOGENE paradigm. In: Proksch HU, Dudeck J, eds. Hospital Information Systems. Amsterdam, The Netherlands: Elsevier Science BV; 1995:347-375.

28. Safran E, Pittet D, Borst F, et al. Alerts as starting point for hospital infection surveillance and control. In: Barahona P, 
Stefanelli M, Wyatt J, eds. Artificial Intelligence in Medicine. Proceedings of the fifth Conference on Artificial Intelligence in Medicine-Europe, AIME 95. Pavia, Italy; June 25-28, 1995. Berlin, Germany: Springer-Verlag; 1995:165-172.

29. Pittet D, Wenzel RP. Nosocomial bloodstream infections: secular trends in rates, mortality, and contribution to total hospital deaths. Arch Intern Med 1995;155:1177-1184.

30. Boyce JM. Incidence of methicillin-resistant Staphylococcus aureus (MRSA) in hospitals in the United States. Infect Control Hosp Epidemiol 1995;16:19. Abstract.

31. Peacock JE, Marsik FJ, Wenzel RP. Methicillin-resistant Staphylococcus aureus: introduction and spread within a hospital. Ann Intern Med 1980;93:526-532.

32. Craven DE, Reed C, Kollisch N, et al. A large outbreak of infections caused by a strain of Staphylococcus aureus resistant to oxacillin and aminoglycosides. Am J Med 1981;71:53-58.

33. Mulligan ME, Murray-Leisure KA, Ribner BS, et al. Methicillinresistant Staphylococcus aureus: a consensus review of the microbiology, pathogenesis, and epidemiology with implications for prevention and management. Am J Med 1993;94:313328.

34. Reboli AC, John JF Jr, Platt CG, Cantey J. Methicillin-resistant Staphylococcus aureus outbreak at a Veterans' Affairs medical center: importance of carriage of the organism by hospital personnel. Infect Control Hosp Epidemiol 1990;11:291-296.

35. Cohen SH, Morita MM, Bradford M. A seven-year experience with methicillin-resistant Staphylococcus aureus. Am J Med 1991;91:233S-237S.

36. Jernigan JA, Titus MG, Gröschel DHM, Getchell-White SI, Farr BM. Effectiveness of contact isolation during a hospital outbreak of methicillin-resistant Staphylococcus aureus. Am J Epidemiol 1996;143:496-504.

37. Walsh TJ, Vlahov D, Hansen SL, et al. Prospective microbiologic surveillance in control of nosocomial methicillin-resistant Staphylococcus aureus. Infect Control 1987;8:7-14.

38. Rao N, Jacobs S, Joyce L. Cost effective eradication of an out- break of methicillin-resistant Staphylococcus aureus in a skilled nursing facility. Infect Control Hosp Epidemiol 1988;9:255-260.

39. Lugeon C, Blanc D, Wenger A, Francioli P. Molecular epidemiology of methicillin-resistant Staphylococcus aureus at a lowincidence hospital over a 4-year period. Infect Control Hosp Epidemiol 1995;16:260-267.

40. McDonald CJ, Hui SL, Smith DM, et al. Reminders to physicians from an introspective computer medical record. A two year randomized trial. Ann Intern Med 1984;100:130-138.

41. McDonald CJ, Tierney WM. Computer-stored medical records. Their future role in medical practice. JAMA 1988;259:34333440.

42. Tate KE, Gardner RM, Waever LK. A computerized laboratory alerting system. MD Computing 1990;7:296-301.

43. McDonald CJ, Tierney WM, Overhage JM, Martin DK, Wilson GA. The Regenstrief medical record system: 20 years of experience in hospitals, clinics and neighborhood health canters. MD Computing 1992;9:206-17.

44. Evans RS, Pestotnik SL, Classen DC, Burke JP. Development of an automated antibiotic consultant. MD Computing 1993;10:1722.

45. Rind DM, Safran C, Philips RS, et al. Effects of computer-based alerts on the treatment and outcomes of hospitalized patients. Arch Intern Med 1994;154:1511-1517.

46. Classen DC, Burke JP. The computer-based patient record: the role of the hospital epidemiologist. Infect Control Hosp Epidemiol 1995:729-736.

47. Hill RLR, Duckworth GL, Casewell MW. Elimination of nasal carriage of methicillin-resistant Staphylococcus aureus with mupirocin during a hospital outbreak. J Antimicrob Chemother 1988;22:377-384.

48. Weems JJ. A plea from the sole: let's keep the 'shoe leather' in healthcare epidemiology. Infect Control Hosp Epidemiol 1996;17:42-43.

\section{Fourth Decennial Conference on Nosocomial Infections, Year 2000}

\section{Gina Pugliese, RN, MS; Martin S. Favero, PhD Medical News Editors}

The CDC has sponsored three previous international conferences on the control of nosocomial infections, in 1970, 1980, and 1990. Plans currently are underway for the newest and largest of these conferences, to be held April 16-19, 2000, at the Marriott Marquis Hotel in Atlanta, Georgia. The Year 2000 conference is sponsored by the $\mathrm{CDC}$ and the
National Foundation for Infectious Diseases (NFID), and is cosponsored by the Association for Professionals in Infection Control and Epidemiology, Inc (APIC), and the Society for Healthcare Epidemiology of America, Inc (SHEA), in cooperation with the American Hospital Association (AHA). The Year 2000 conference will be held in conjunction with SHEA's 10th Annual Meeting in the year 2000.

The Year 2000 Coordinating Committee, chaired by the director of the CDC's Hospital Infections Program, with representatives from NFID, APIC, SHEA, and AHA, met recently at the $\mathrm{CDC}$ in Atlanta to plan the conference. It is estimated that 2,000 individuals will attend. For the first time, there will be commercial exhibits at the conference. Registration, the opening session, and a reception are scheduled for Saturday, April 15, and plenary, concurrent, and poster sessions will be held through Wednesday, April 19, 2000. Publicity plans are being made. 